\title{
Jean-Joseph Rabearivelo y el mundo hispánico
}

\author{
Guillermo Pié Jabn \\ Universitat Rovira i Virgili \\ guillermo.pie@urv.cat \\ Irina Razafimbelo \\ Université d'Antananarivo \\ razaf79@hotmail.com
}

Resumen: Jean-Joseph Rabearivelo (Antananarivo, 1901-1937) es el escritor más importante de Madagascar y uno de los autores africanos francófonos más reputados y universales. Además de en malgache y en francés, Rabearivelo escribió poesía en español, décadas antes de que naciera la literatura ecuatoguineana. Por ello, podemos considerarlo el primer escritor contemporáneo en lengua española del África subsahariana. Tradujo a Góngora al idioma malgache, por lo que fue también el primer traductor de textos literarios españoles a una lengua vernácula africana.

Palabras clave: Rabearivelo, Madagascar, África, Español como lengua extranjera, Literatura, Alfonso Reyes, Góngora.

Abstract: Jean-Joseph Rabearivelo (Antananarivo, 1901-1937) is the most important writer of Madagascar and one of the most renowned and universal African French-speaking authors. Besides writing in Malagasy and French, Rabearivelo also wrote poetry in Spanish, decades before the Equatoguinean literature appeared. Therefore, we can consider him the first contemporary writer in Spanish language of the Sub-Saharan Africa. He translated Góngora to the Malagasy language, so he was also the first translator of literary Spanish texts to a vernacular African language.

Key words: Rabearivelo, Madagascar, Africa, Spanish as a foreign language, Literature, Alfonso Reyes, Góngora. 


\section{Introducción}

Jean-Joseph Rabearivelo, el príncipe de los poetas malgaches —así lo llamó Léopold Sédar Senghor en su famosa antología de 1948 (Riffard, 2007: 110)—, nació en el barrio Isoraka de Antananarivo, capital de Madagascar, el 4 de marzo de $1901^{1}$. Su familia, perteneciente a la casta noble de los Zanadralambo, se empobreció a raíz de los cambios introducidos por la colonización francesa ${ }^{2}$. Rabearivelo abandonó tempranamente la escuela, en 1916. Se ocupó en diversos oficios: secretario, diseñador textil, bibliotecario, hasta que en 1924 entró a trabajar como corrector en la editorial malgache Imerina, puesto de baja remuneración que mantendría hasta su muerte. Fue un escritor precoz: a la edad de 14 años ya había publicado sus primeros poemas en lengua malgache ${ }^{3}$.

En 1920 conoció a Pierre $\mathrm{Camo}^{4}$, poeta y magistrado francés que influiría mucho en su obra primeriza, muy marcada por los modelos parnasianos y simbolistas franceses. Durante esta primera época, que abarca toda la década de los años 20, escribió diversos poemarios en lengua francesa, de los que sobresalen La coupe de cendres (1924), Sylves (1927) y Volumes (1928). También escribió, entre otras obras narrativas, dos novelas ambientadas en las postrimerías de la monarquía merina y los primeros años de la colonización: L’aube rouge (1925) y L'interférence (1928), ambas publicadas póstumamente.

En los años 30 Rabearivelo buscó un estilo más personal y enraizado en la cultura malgache, aunque también fue permeable a las nuevas corrientes literarias que venían del exterior, en especial el surrealismo. Durante estos años, Rabearivelo escribió diversos poemarios en verso libre. Los más conocidos son los poemarios bilingües malgache-francés Saika-nofy/Presque-songes (1934) y Nadika tamin'ny alina/Traduit de la nuit (1935)5. Asimismo, con la idea de recuperar y preservar el folklore de Madagascar, Rabearivelo cultivó el «hain-teny» [«ciencia de la palabra» $\mathrm{o}$ "palabras sabias»], forma tradicional de la poesía oral malgache. También escribió obras de teatro como Imaitsoanala, zana-borona/Imaitsoanala, fille d'oiseau (1935) y Aux portes de la ville (1936).

Amén de poeta, narrador y dramaturgo, Rabearivelo fue un fecundo diarista, crítico literario y traductor. Su actividad epistolar fue también muy intensa: llegó a cartearse con figuras como Paul Valéry o André Gide. Recientemente, hemos

\footnotetext{
1 Los estudiosos de su obra tienen dudas sobre si nació en 1901, 1903 o 1904 (Joubert, 1991).

2 Madagascar fue colonia francesa desde 1896 hasta 1960.

3 En la revista malgache Vakio ity [Lee esto].

4 Pierre Camo (1877-1974). Poeta francés. Fue magistrado en Madagascar desde 1903 hasta 1934.

5 Ambos poemarios han sido traducidos al español por Juan Abeleira para la editorial Hiperión (V. Rabearivelo, 2000).
} 
hallado en México varias cartas inéditas ${ }^{6}$ que Rabearivelo envió a uno de los intelectuales más importantes de las letras hispanas del siglo xx: Alfonso Reyes. El contacto epistolar con Alfonso Reyes se inició en 1932, cuando éste se hallaba destinado como embajador de México en Brasil. La correspondencia con el autor de Cuestiones gongorinas se mantuvo durante años y fue para el joven poeta malgache una ventana abierta al mundo literario en español.

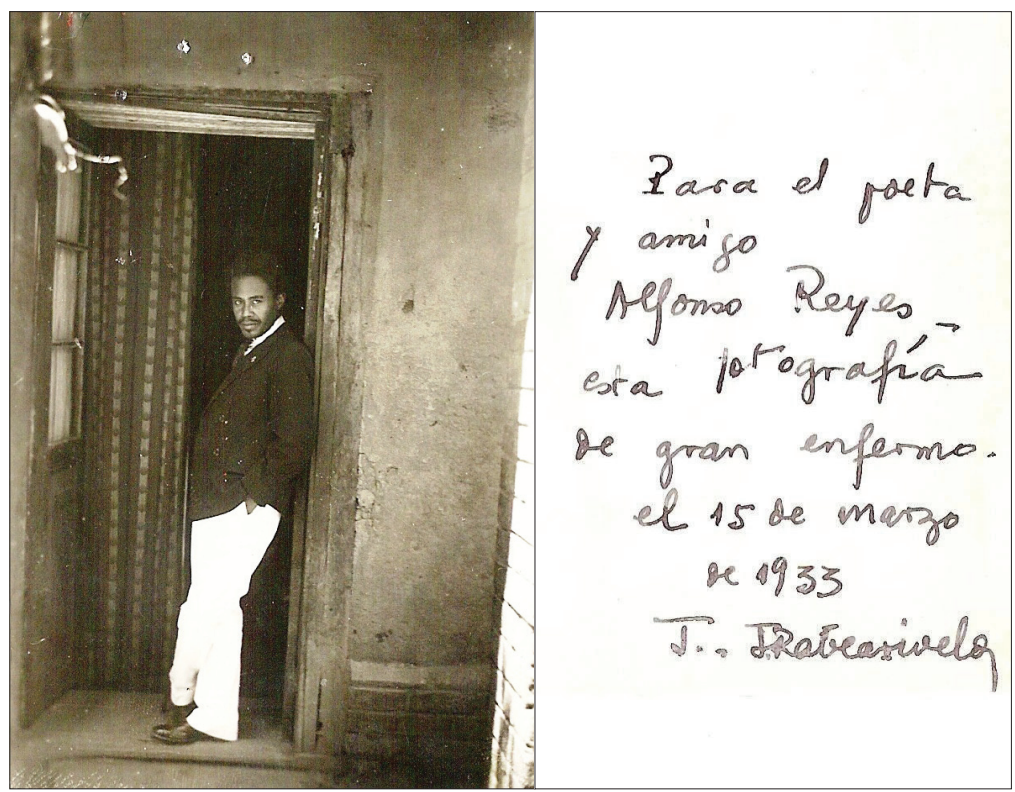

Fotografía inédita enviada por Jean-Joseph Rabearivelo a Alfonso Reyes en 1933. El texto está en el reverso. Por gentileza de la Dra. Alicia Reyes, nieta del escritor mexicano.

El 22 de junio de 1937, Rabearivelo, aquejado de una grave depresión y sintiéndose alienado entre dos culturas, decidió recurrir al suicidio y reunirse definitivamente con los ancestros. Murió como quiso vivir: escribiendo. Poco antes de ingerir diez gramos de cianuro potásico, Rabearivelo anotó estas palabras en su diario Les calepins bleus:

Ce n'est pas drôle : un latin parmi les Welches, et avec les traits d'un Welche - ceci soit dit sans moquerie aucune : Imaginez, en renversant les rôles, Jésus Européen, (origine, traits, etc.).

6 El corpus, propiedad de la familia de Alfonso Reyes, se compone de 14 folios manuscritos y esta foto con texto en el reverso. Hay, además, algunos recortes de periódico, una tarjeta y varias cartas que intercambiaron Reyes y su mujer con Solofo, el hijo mayor de Rabearivelo. 
Et cela, c'est moi : impérieusement, violemment, naturellement latin chez les Mélaniens. Et avec les traits de ceux-ci.

Non, ça ne peut pas continuer ainsi. ${ }^{7}$

\section{Rabearivelo, traductor de Góngora al idioma malgache}

Rabearivelo se mostró siempre muy atraído por los problemas de la traducción poética. Su proyecto de crear una literatura malgache moderna pasaba por verter a su lengua materna una selección de obras extranjeras ejemplarizantes. En este contexto hay que situar las traducciones que realizó de uno de los poetas más representativos del barroco español: Luis de Góngora. Rabearivelo profesó por este escritor una profunda veneración: las Soledades era uno de sus libros de cabecera y guardaba en su modesta casa de Antananarivo un busto del poeta cordobés al que compuso varios poemas.

Tal y como ha explicado su hijo Solofo, Rabearivelo comenzó a estudiar el español para poder leer a Góngora en su versión original. Autodidacta constante y pertinaz, se entregó con entusiasmo a la excéntrica tarea de aprender español en el Madagascar de los años 20 y 30. Lo hizo solo: leyendo, escribiendo, traduciendo durante años, a golpe de diccionario y a la luz de un quinqué. Como él mismo decía en su diario inédito Les calepins bleus: «Espagne, terre inconnue... La decouvrir pour soi, au prix de quel travail $\left.+_{+}\right\rangle^{8}$.

En una de las cartas inéditas que hemos descubierto en México, fechada el 25 de mayo de 1933, Rabearivelo revela a Alfonso Reyes que está trabajando en la traducción de las Soledades al malgache. No tenemos noticia de que esa traducción llegara a ser publicada. En cambio, sí hemos hallado tres sonetos de Góngora traducidos al malgache ${ }^{9}$. Fueron publicados en 1932 en la gaceta de Antananarivo Ny Fandrosoam-baovao [El nuevo progreso].

Sobre estas traducciones Alfonso Reyes escribió en 1933 una breve reseña en el Boletin Gongorino de su famosa revista Monterrey (Reyes, 1933: 4):

\section{Góngora EN MALGACHO}

El poeta J.-J. Rabearivelo (conocido ya de los lectores del Journal des Poètes, Bruselas) publica en el Fandrosoam-Baovao, comentario sobre Góngora, al que pone en el triángulo de la poesía hermética con el italiano Marini y el

\footnotetext{
7 De su diario inédito Les calepins bleus, 22 de junio de 1937. Agradecemos a la Dra. Riffard habernos permitido acceder a distintos fragmentos del mismo.

8 Ibidem, 27 de febrero de 1933.

9 Ny Fandrosoam-baovao, no 53, Antananarivo, septiembre de 1932.
} 
francés Maurice ScÈve, y traduce al malgacho los tres sonetosः «Descaminado, enfermo, peregrino», «Tras la bermeja aurora el sol dorado» e «Ilustre y hermosísima señora». El poeta de Madagascar declara haberse sentido atraído por la lengua de Góngora porque cree hallar en su fonética una alianza del francés con el malgacho. Señalamos a los curiosos este expresivo ejemplo de la difusión de nuestras letras y de nuestro Don Luis.

Les ofrecemos a continuación esos tres sonetos de Góngora — nada menos que Góngora- en lengua malgache, publicados en septiembre de 1932 y que hemos conseguido encontrar no sin esfuerzo. Constituyen, como hemos dicho, las primeras traducciones de textos literarios españoles a una lengua africana. I.

Very làlana, marary ary irery an-tanin'olona, misolafaka hatrany, ao anaty alina matevina, mamindra eo amin'ny fisafotofoton'ny efitra mangina, io izy miantsoantso foana ary mandroso tsy manan-kaleha.

Injay reny miavaka any, na dia mbola lavitra aza, ny voron'ny amboa tsy mitsaha-miambina ; ary tao ambany tafon'ny tranon-tantsaha iray, dia tojo fo mangoraka izy, na dia very làlana aza.

Niposaka ny masoandrao, nisaly volombiby fotsy, ary tamin'ny heribakoran'ny hasoany valabalaka dia nanaitra ilay mpivahiny izay efa nilaozan-kery.

Ny ainy no hanefa ity fandrosoam-bahiny ity.

Metimety ho azy kokoa raha niriorio tany an-tendrombohitra izy, tsy izay ho faty toy izany fahatsiarovako ahy ho faty izany.

II.

Ery amin'ny varavaran'ny Atsinanana, aorian'ny vilaory tinafin'ny terak'andro, io mipoitra ny masoandro volamena : ny iray mapihaingo voninkazo ny handriny mavokely, ny iray kosa mampisatroka azy afo mazava.

Amin-peo malefaka na amin-peo mitalaho, eo an-tanimbilona maitso, eo amin'ny hamandomandon'ny rivotra, eo anatrehan'ny andro terabao, ny vorona mahafinaritra dia mitango ny hasambarana amanalahelony.

Amin'izay, mivoaka ny tranonandrianany, io Leonora manolotra ny tavany amin'ny rivotra ary mihira ka manome fanahy hatraminny vato. Amin'izay,

dia tsy reko intsony ny vorona, tsy hitako intsony torak'andro, fa raha vao tonga izy dia tankim-peo sy tapi-porohana ny rehetra, ary zary jamba sy marenina ho azy rehetra aho.

III.

Ry Mariako miendriḱandriana sy soa indrindra, raha mbola apetraky ny ora hatrany eo amin'ny endrika-voninkazonao ny raozin'ny terakandro, ary eo amin'ny handrinao sy ny masonao ny Masoandro,
I.

Descaminado, enfermo, peregrino, en tenebrosa noche, con pie incierto la confusión pisando del desierto, voces en vano dio, pasos sin tino.

Repetido latir, si no vecino, distinto, oyó de can siempre despierto, y en pastoral albergue mal cubierto, piedad halló, si no halló camino.

Salió el Sol, y entre armiños escondida, soñolienta beldad con dulce saña salteó al no bien sano pasajero.

Pagará el hospedaje con la vida; más le valiera errar en la montaña que morir de la suerte que yo muero.

II.

Tras la bermeja Aurora el Sol dorado por las puertas salía del Oriente, ella de flores la rosada frente, él de encendidos rayos coronado.

Sembraban su contento o su cuidado, cuál con voz dulce, cuál con voz doliente, las tiernas aves con la luz presente en el fresco aire y en el verde prado.

Cuando salió bastante a dar Leonora cuerpo a los vientos y a las piedras alma, cantando de su rico albergue, y luego

ni oí las aves más, ni vi la Aurora; porque al salir, o todo quedó en calma, o yo (que es lo más cierto), sordo y ciego.

III.

Ilustre y hermosísima María, mientras se dejan ver a cualquier hora en tus mejillas la rosada Aurora, Febo en tus ojos y en tu frente el día, 
raha mbola mitopa ny rivotra ka misangy malefaka ny hango ankatokao izay toa voahaingo amin'izay ireharehan'i Arabia manontolo, ary ny ony Tajo mbola mipoitra avy amin'ny fasika ;

dieny mbola tsy takona ny dian'ny Masoandro, ary tsy mbola miofo ho ali-maizina ny andro mamiratra, satria ny terak'andro mbola mahadify ny rahom-pahafatesana ;

dieny mbola eo aminao ny tahiry mivolom-bola, mangatsakatsaka noho ny orampanala mangatsaka, otoazy re, otoazy ny endrika sy ny hazavana ary ny volamena. y mientras con gentil descortesía mueve el viento la hebra voladora que la Arabia en sus venas atesora y el rico Tajo en sus arenas cría; antes que, de la edad Febo eclipsado y el claro día vuelto en noche obscura, huya la Aurora del mortal nublado;

antes que lo que hoy es rubio tesoro venza a la blanca nieve su blancura: goza, goza el color, la luz, el oro.

\section{Rabearivelo, primer escritor contemporáneo del África subsahariana en lengua española}

Como hemos dicho, amén de en francés y malgache, Rabearivelo compuso también poesía en español, décadas antes de que naciera la literatura de Guinea Ecuatorial ${ }^{10}$. En este sentido, Rabearivelo puede ser considerado el primer escritor contemporáneo del África subsahariana en lengua española.

Dado que Rabearivelo quemó la parte de su diario íntimo correspondiente a los años 1924-1933, no sabemos con precisión cuándo comenzó su aprendizaje del español. La correspondencia recién hallada en México nos revela que con toda seguridad en una fecha anterior a 1932, pues en una carta fechada el 27 de noviembre de ese año Rabearivelo comienza dirigiéndose a Alfonso Reyes en español para después confesarle con pudor en francés que es un autodidacta de la lengua española y que incluso ha escrito, en un tiempo no muy lejano, versos en español.

En otra carta, de 15 de marzo de 1933, Rabearivelo explica a Reyes que, convaleciente de una grave enfermedad ${ }^{11}$, ha decidido retirarse al campo, donde piensa releer las Soledades de Góngora y escribir un poemario en español titulado Ventanas, compuesto por «quebrados de música sorda».

Posteriormente, en una carta a su amigo Robert Boudry, transcrita a su diario íntimo el 11 de septiembre de 1935, pero sin duda anterior a esa fecha, aludirá a la escritura de dos poemarios en lengua española: Ventanas abiertas y Vientos cautivos. En su diario también mencionará en otras ocasiones el poemario Vientos cautivos.

Sean diferentes poemarios o uno solo en distintas etapas de elaboración, lo cierto es que a mediados de los años 30 Rabearivelo tenía escrito en español un poemario titulado Vientos de la mañana. Así se desprende de las repetidas alusio-

10 La primera novela ecuatoguineana se publicó en 1953. Las primeras muestras de poesía aparecieron más tarde, a mediados de los años 60 (Ndongo Bydiogo y Mbaré, 2000).

11 Rabearivelo era asmático. 
nes que hay en su diario y así lo atestiguaron en su día dos de sus amigos íntimos, Robert Boudry ${ }^{12}$ y el reconocido escritor malgache Jacques Rabemananjara:

Il fait souvent dans son Journal des citations en espagnol, lit les auteurs et en particulier Góngora car tout ce qui est préciosité et hermétisme l'intéresse. Il réunit dans la même pensée Góngora, Marini et Mallarmé. Il possède suffisamment l'espagnol pour écrire des poèmes et les publier dans cette langue, sous le titre Vientos de la Mañana (Boudry, 1958: 45).

Du surcroît, il a appris l'espagnol au point d'avoir pu composer un grand poème dans l'idiome de Cervantes, intitulé Los Vientos de la mañana. Il ne máa jamais montré le manuscrit ; mais certaines correspondances qu'il entretenait avec des hispanisants y faisaient une allusion élogieuse (Rabemananjara, 1989: 33).

Solofo, el hijo mayor de Rabearivelo, anduvo buscando este poemario durante varias décadas, para lo cual contó incluso con la colaboración de Alfonso Reyes (Reyes, 1956: 650-651). Sin embargo, nunca pudo dar con él. Vientos de la mañana, como tantos otros escritos de Rabearivelo, sigue desaparecido.

A finales de 1936, pocos meses antes de su suicidio, Rabearivelo escribió en un poemario, todavía hoy inédito, llamado Proses pour Durtal, un largo poema titulado Le triple chiffre ${ }^{13}$. El poema va dedicado a Alfonso Reyes y Armand Guibert $^{14}$, e incluye un pasaje escrito en lengua española. Mientras no aparezcan los poemarios antes señalados, este pasaje constituye la única muestra conservada de la poesía de Rabearivelo en español.

En Le triple chiffre, que transcribimos a continuación, Rabearivelo dirige un alienado apóstrofe a la ciudad de Antananarivo, a la que imagina como una gitana española; habla de sus recientes lecturas en español ${ }^{15}$ : el Romancero Viejo, el Romancero Gitano de Federico García Lorca - que acababa de ser fusilado- y Bajo los ángeles de Rafael Alberti; confiesa su amistad espiritual hacia estos dos poetas de la Generación del 27 y su amor por las tres culturas: la malgache, la francesa y la española.

He aquí el poema en cuestión:

12 Robert Boudry (1893-1958). Escritor francés. Fue director financiero de la colonia de Madagascar y, posteriormente, gobernador de los territorios franceses de ultramar.

$13 \mathrm{El}$ poema fue incluido como anexo en Nonn, 1998.

14 Armand Guibert (1906-1990). Escritor, editor y traductor francés. Editó la obra de Rabearivelo Traduit de la nuit en 1935.

15 Aparte de clásicos como Góngora y Quevedo, en su diario y su correspondencia aparecen frecuentemente referencias a lecturas de autores hispanohablantes coetáneos: Alfonso Reyes, Ortega y Gasset, Eugenio d'Ors, García Calderón y, amén de Lorca y Alberti, otros poetas del 27 como Salinas. 
LE TRIPLE CHIFFRE

Pour Alfonso Reyes et Armand Guibert

Nul âne ne paresse en ce paysage

que brûle et désole le soleil

nul âne nulle mule

les taureaux on ne les y fête plus

pour la joie ivre de les voir mourir

et rien de ce qui fit dire à Victor Hugo

que Besançon était une vielle ville espagnole

ne mautoriserait non plus mon Iarive ${ }^{16}$

à faire de toi une Castillane

Or pourquoi mobstiné-je

à voir en toi un autre Orient dans l'Occident

tout comme cette péninsule ibéro-mauresque

que j'aime tant pour sa profondeur

Est-ce parce qu'un panier de crevettes roses

et de citrons et d'autres primeurs des côtes

est là

devant moi

sur l'herbe maigre de cette cime jumelle

où je me surprends

à te désirer de loin

Est-ce parce que le printemps fleurit ton ciel

tandis que ton front est fouetté par la pluie

ou bien parce que dans l'ardeur de la nuit

hier

je me suis enivré en me récitant

tant de romances viejos

dont celui d'Abenamar

mais plus má plu celui de la Rosa fresca

et les poèmes d'aujourd'hui

de l'angélique Alberti

et de Lorca notre Federico le massacré de septembre

Mais n'est-ce pas plutôt parce que la Terre

n'ayant plus de secrets

et nétant plus que la terre

16 Nombre poético de la ciudad de Antananarivo. 
grâce au progrès

les hommes sont plus nus et misérables

et plus miséreux

et qu'habitant des maisons de cristal

et nantis de corps eux-mêmes translucides

ils n'ont plus rien à se cacher

et que se perçoit

presque simultanément

de l'un à l'autre hémisphère

jusqu'au cœur qui bat

et aux cils qui tremblent

Et de là-bas c'est tout si nous nentendons pas

aujourd'hui

avec la vitesse de la lumière

jusqu'au bruit de leurs canons

qui saccagent leurs propres annones

Mais le printemps est sur ton ciel

mon Iarive

et bourgeons et fleurs tissent des couronnes

autour de ton front que bat la pluie

et que cache le brouillard

Ah ne pensons plus aux dernières ondes

dont chaque oscillation nous fait vaciller le cœur

Ecoute

Ecoute

dans cet hispano-français

quà force d'ignorance et d'amour

je me suis forgé

ce chant malhabile et passionné

qui te va mieux que tout autre

en cet instant où je te débaptise

et t'imagine un peu gitane

Donde estas amiguita mia que no conozco

y que no obstante me conoces

y murmuras mi nombre

Donde estas hermosa que nunca jamas he visto

y que no obstante duermes en mi cabeza 
$y$ tienes

sueños en mi corazon

Donde

donde estas

Et c'est toi cette belle inconnue

que j'eusse aimée et qui le savait

El c'est dans l'amitié spirituelle

de deux Andalous

l'un Gaditan et l'autre Grenadin

que je vous dédie éperdument

le rythme celé de cette presque-romance

où se retrouve le triple chiffre obscur

dont vous marquez votre amant

Iarive mon Iarive

ô mon tombeau

et toi ma française lyre

que dans mon cœur j’ai taillé

et toi España inmortal

terre plus double que la mienne

Numerosos investigadores han tratado la alienación y el desgarramiento cultural que padeció Rabearivelo a causa de la colonización ${ }^{17}$. Se sentía dividido entre dos mundos: el de los suyos y el de aquellos que colonizaron su tierra. Incomprendido y maltratado por ambas partes, Rabearivelo gestó durante años una crisis personal que lo condujo finalmente al suicidio. Algunos estudiosos de su obra no han dudado en calificar su muerte de suicidio colonial $^{18}$.

Sin embargo, había en Rabearivelo un tercer componente obviado por la crítica hasta ahora: su relación con el español y la cultura hispánica. La aproximación de Rabearivelo a la lengua española fue una elección entusiasta, libre y personal: «Je songe aussi à la gratuité donc à la pureté absolue du plaisir fourni par une acquisition intellectuelle de plus» ${ }^{19}$. Lo que comenzó como un juego intelectual ${ }^{20}$ fue, sin embargo, cobrando con el tiempo fuerza y sentido, hasta el punto de que, en su momento de plenitud y genialidad literaria, llegó a combinar la escritura no

17 También influyeron otras circunstancias como la muerte de su hija Voahangy en 1933, las dificultades económicas, su estado enfermizo... Un hecho determinante fue que la administración colonial no le permitiera representar a Madagascar en la Exposición Internacional de París en 1937.

18 V. Boudry, 1958; Rabemananjara, 1989; Schild, 1990; Joubert, 1991.

19 De su diario inédito Les calepins bleus, 27 de febrero de 1933.

20 Rabearivelo se inició someramente en el estudio de otras lenguas como latín o esperanto. 
en dos sino en tres lenguas (malgache, francés y español) ${ }^{21} y$, como hemos visto en Le triple chiffre, a confesar abiertamente su triple filiación cultural.

\section{Conclusión}

Jean-Joseph Rabearivelo, el gran poeta de Madagascar, fue el primer escritor contemporáneo en lengua española del África subsahariana y el primer traductor de textos literarios españoles a una lengua vernácula africana.

Rabearivelo se suicidó joven, cuando su fascinante aventura literaria en español apenas había comenzado. Hay textos perdidos y muchos interrogantes todavía, pero con el presente trabajo ${ }^{22}$ hemos querido rendir un homenaje a este poeta universal y recuperar del olvido unas cartas, unos versos, unas traducciones que tienen el valor añadido de ser patrimonio común de la cultura malgache y la cultura hispánica.

\section{Referencias bibliográficas}

Adejunmobi, M. (1996). JJ Rabearivelo, Literature and Lingua Franca in Colonial Madagascar. Nueva York. Lang.

Boudry, R. (1958). Jean-Joseph Rabearivelo et la mort. París. Présence Africaine. Joubert, J.-L. (1991). Littératures de l'Océan Indien. Vanves. EDICEF.

Ndongo Bydiogo, D. y Mbaré, N. (eds.) (2000). Literatura de Guinea Ecuatorial (Antología). Madrid. Sial.

Nonn, E. (1998). Imerina. París. Verticales.

Rabearivelo, J.-J. (1924). La coupe de cendres. Antananarivo. G. Pitot de la Beaujardière.

- (1927). Sylves. Antananarivo. Imprimerie de l'Imerina.

- (1928). Volumes. Antananarivo. Imprimerie de l'Imerina.

- (1934). Presque-songes. Antananarivo. Imprimerie de l'Imerina.

- (1935). Traduit de la nuit. Túnez. Éditions de Mirages.

- (1935). Imaitsoanala, fille d'oiseau. Antananativo. Imprimerie officielle.

- (1936). Aux portes de la ville. Antananarivo. Imprimerie officielle.

- (1988). L’Interférence. París. Hatier.

- (1998). Laube rouge. París. Omnibus.

21 Rabearivelo escribió Vientos de la mañana en la misma época que sus obras maestras Saika-nofy/Presquesonges (1934) y Nadika tamin'ny alina/Traduit de la nuit (1935).

22 Esta investigación se prolongó durante varios años, desde 2004 hasta 2008. Tenemos previsto reanudarla si aparecen nuevos documentos que relacionen a Rabearivelo con la lengua española. 
- (2000). Casi sueños. Traducido de la noche. Madrid. Hiperión.

Rabemananjara, J. (1989). "50ème Anniversaire de la mort de Jean-Joseph Rabearivelo». En: Jean-Joseph Rabearivelo, cet inconnu? Colloque International de l'Université de Madagascar. Marsella. SUD.

Reyes Ochoa, A. (1933) (ed.). «Góngora en malgacho». Monterrey, Correo literario de Alfonso Reyes, $\mathrm{n}^{\circ}$ 10, Boletín gongorino V, Río de Janeiro, Apollo, p.4.

- (1956). «Un gongorino en Madagascar». Obras Completas (1989). México. Fondo de Cultura Económico, vol. XXII: 648-651.

Riffard, C. (2007). «Jean-Joseph Rabearivelo. Prince des poètes malgaches». Cultures Sud, no 164. París. Institut Français: 110-123.

Schild, U. (1990). «Der Gesang der Vogeltochter oder der Versuch, den Freitod des Dichters Jean-Joseph Rabearivelo kulturell zu deuten». En: KoнL, K.-H.; Muszinski, H. y Strecker, U. (eds.). Die Vielfalt der Kultur: Ethnologische Aspekte von Verwandtschaft, Kunst und Weltauffassung. Berlín. Reimer: 466478.

Senghor, L. S. (ed.) (1948). Anthologie de la nouvelle poésie nègre et malgache de langue française. París. Presses Universitaires de France. 